\title{
Kinetic modeling of the electronic response of a dielectric plasma-facing solid
}

\author{
Franz X. Bronold and Holger Fehske \\ Institut für Physik, Ernst-Moritz-Arndt-Universität Greifswald, 17489 \\ Greifswald, Germany \\ E-mail: bronold@physik.uni-greifswald.de
}

\begin{abstract}
We present a self-consistent kinetic theory for the electronic response of a plasma-facing dielectric solid. Based on the Poisson equation and two sets of spatially separated Boltzmann equations, one for electrons and ions in the plasma and one for conduction band electrons and valence band holes in the dielectric, the approach gives the quasi-stationary density and potential profiles of the electric double layer forming at the interface due to the permanent influx of electrons and ions from the plasma. The two sets of Boltzmann equations are connected by quantum-mechanical matching conditions for the electron distribution functions and a semi-empirical model for hole injection mimicking the neutralization of ions at the surface. Essential for the kinetic modeling is the ambipolarity inside the wall, leading to an electron-hole recombination condition, and the merging of the double layer with the quasi-neutral, field-free regions deep inside the wall and the plasma. To indicate the feasibility as well as the potential of the approach we apply it to a collisionless, perfectly absorbing interface using intrinsic and extrinsic silicon dioxide and silicon surfaces in contact with a two-temperature hydrogen plasma as an example.
\end{abstract}

PACS numbers: 52.40.Hf, 52.40.Kh, 68.49.Jk, 68.49.Sf 


\section{Introduction}

The basic electronic response of a plasma-facing solid (wall) is the accumulation of electrons from the plasma. It acquires a negative charge because impacting electrons are deposited inside the solid more efficiently than electrons are extracted from it by the neutralization of positive ions. The negatively charged wall in turn triggers a positively charged space charge region in front of it: the plasma sheath [1, 2]. The total result of the electronic response of the plasmawall interface is thus an electric double layer. Yet, ever since the early days of gaseous electronics [3], the negative part of the double layer-the wall charge-plays no essential role in the studies of the plasma sheath. Typically the focus is either on how the sheath merges with the quasi-neutral bulk plasma (see the reviews 4 , 5, 6, 7, 8, and references therein) or on how the emissive properties of the wall, most notably, electron reflection (or absorption) and secondary electron emission, affect the spatial structure of the sheath [9, 10, 11, 12, 13.

Evidently for this type of studies the wall is considered as a reservoir characterized by a geometrical boundary and probabilities for particle reflection, absorption, and emission which may be chosen adhoc to make plasma simulations reproduce experimental findings, deduced from quantum-mechanical calculations [14, 15], or-in very rare occasions-obtained from independent measurements [16, 17. In situations where the length and time scales of the plasma and the wall are well separated this is a viable strategy. Even microdischarges [18] with a linear extension of $15-40 \mu \mathrm{m}$ can be successfully modeled by such an approach [19. However, if this is not the case, or if the electrons accumulated in the solid are an integral part of the physical system one is interested in, as it is, for instance, the case for the plasma bipolar junction transistor [20, this approach is not sufficient. A kinetic description has then to be set up also for the wall and merged to the one of the plasma by suitable matching conditions.

In particular, solid-state based integrated microdischarges [21, 22, 23, 24] can be expected to soon reach the sub-micron range where the electron transit time $\tau_{e}^{\text {transit }}$ through the sheath of the discharge approaches the electron energy relaxation time $\tau_{*}^{\text {relax }}$ inside the solid. In this case, the electronic subsystem of the wall remains out-of-equilibrium between subsequent electron encounters from the plasma and surface parameters have to be obtained for a solid in strong electronic non-equilibrium. Taking, as an illustration, a $1 \mu \mathrm{m}$ wide microdischarge with a screening length $\lambda_{D}^{p}$ of one-tenth of its width, which seems to be feasible [24, and an electron temperature $k_{B} T_{e}=$ $2 \mathrm{eV}$ yields $\tau_{e}^{\text {transit }} \approx \lambda_{D}^{p} / \bar{v}_{e} \approx 10^{-13} \mathrm{~s}$, where $\bar{v}_{e}=$ $\sqrt{8 k_{B} T / \pi m_{e}}$ is the thermal velocity of the electron. This is only one order of magnitude smaller than the typical electron energy relaxation time in the conduction band of a dielectric solid such as $\mathrm{SiO}_{2}, \tau_{*}^{\text {relax }} \approx$ $l_{\text {inel }} / v_{*} \approx 10^{-14} \mathrm{~s}$, assuming a kinetic energy of the injected electron of $2 \mathrm{eV}$ above the bottom of the conduction band, an effective mass of $0.8 m_{e}$, and an inelastic scattering length of $l_{\text {inel }} \approx 100 \AA$ (from the universal curve [25]), but already much shorter than the electron-hole recombination time which is on the order of nanoseconds. In addition, the separation between integrated microdischarges can be made small enough to enable crosstalk through the space charge layers inside the wafer opening thereby perhaps opportunities for novel opto-electronic plasma devices. There are thus situations conceivable where the electronic processes in the solid and the plasma cannot be considered independently anymore. Focusing on the positive part of the electric double layer alone will then be of course also no longer sufficient.

Double layers are abundant in nature and have been studied in various contexts. They arise at any interface separating positive and negative charges. In solid state physics the most important double layer is the pn-junction [26] which is at the heart of many electronic devices. Double layers occur also when two different gaseous plasmas approach each other [27, 28, 29,30 . The double layer at the plasma-wall interface is however special. On the one hand, and in contrast to pn-junctions, it is always far from equilibrium, involving very hot charge carriers. On the other hand, and this distinguishes it from the gaseous double layers, it is spatially pinned by the crystallographic ending of the wall and energetically constrained by the wall's band structure. Irrespective of the demands arising from the miniaturization of microdischarges studying double layers at plasma-wall interfaces is thus also of fundamental interest.

Up to now electric double layers at plasma-facing solids have not been studied extensively. To the best of our knowledge metallic surfaces have not been investigated at all and there are only a few studies 31 , 32 devoted to dielectric surfaces in contact with a plasma. But even for them a satisfactory description is still missing. In our own work 32 on the subject, for instance, we employed a thermodynamical principle to distribute the electrons missing in the plasma sheath in a graded potential which interpolates between the sheath and the potential inside the wall [32. The basic assumption, however, that at quasi-stationarity the electrons are thermalized within the wall, is only valid for some of the electrons and not for all. In addition, the approach was based on drift-diffusion equations. Hence, the dynamical variables were from the start particle densities, fluxes and electric 
potentials. Distribution functions did not appear. The coupling of the positive and negative parts of the double layer was thus entirely due to the matching conditions for the electric potential. It was hence impossible to include quantum-mechanical reflection of electrons by the surface potential and/or electron extraction due to the neutralization of ions.

Below we present a kinetic approach for a dielectric surface which is general enough to overcome these shortcomings. It works with the Poisson equation and two sets of Boltzmann equations operating in disjunct half-spaces. The matching at the interface is performed not only for the electric potential but also for the distribution functions which enables us to keep the ambipolarity of the plasma side (electrons and ions) alive inside the wall (electrons and holes). Eventually, this allows us to formulate a recombination condition for electrons and holes, which in turn limits, in conjunction with further conditions imposed at quasi-stationarity, the continuous influx of electrons and ions from the plasma. For the electron distribution functions the matching conditions are essentially identical with the matching conditions used for solid interfaces [33, 34, 35]. The matching condition for the ion distribution function on the other hand is a semiempirical model for electron extraction (that is, hole injection) connecting the ion distribution function of the plasma with the hole distribution function of the wall. A thermodynamical principle is no longer used. Instead, we only demand as boundary conditions quasineutral, field-free regions far away from the interface.

In the numerical calculations we consider a collisionless perfectly absorbing surface. But the kinetic approach is first described in broader terms so that it becomes clear how to include collisions and how to include quantum-mechanical reflection of electrons. The numerical solution of the complete kinetic model is however rather demanding and beyond the scope of the present work. The numerical calculations yield thus only the self-consistent quasi-stationary potential and density profiles across an idealized interface. For given temperature and mass ratios the incoming flux of electrons and ions is self-consistently determined. Despite the simplicity of the model, the numerical results for $\mathrm{SiO}_{2}$ and $\mathrm{Si}$ surfaces in contact with a hydrogen plasma are very promising and clearly indicate the feasibility and potential of our approach for revealing the rich physics taking place inside the wall.

The outline of the remaining part of the paper is as follows. In Sect. 2 we describe in detail our approach, first, in general terms and then for the special case of a collisionless, perfectly absorbing dielectric surface. Section 3 discusses representative numerical data for idealized intrinsic and doped

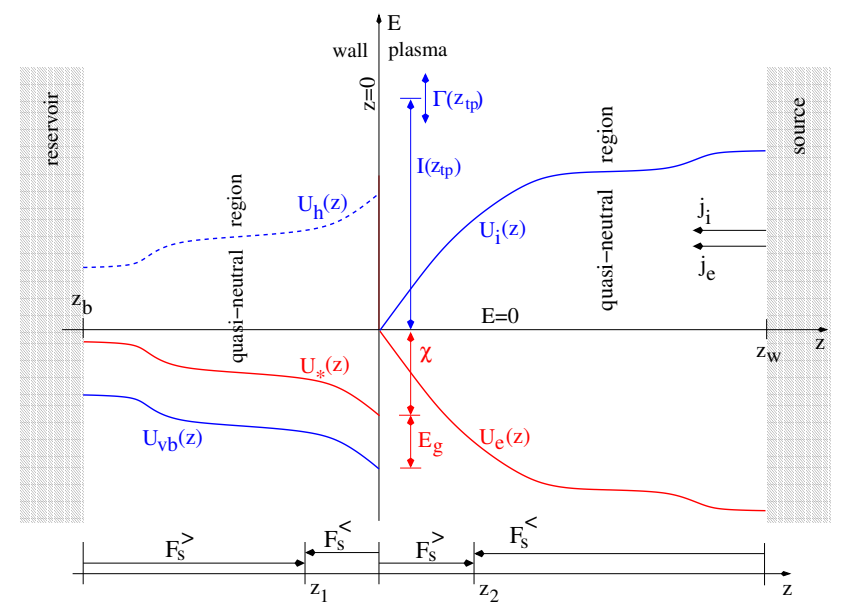

Figure 1. (Color online) Illustration of the interface model on which our calculation is based anticipating an electric double layer with negative space charge inside the wall and positive space charge in front of it. We consider a planar interface at $z=0$ separating a dielectric wall from a plasma. The wall contains two bands, an initially fully occupied valence band and an initially empty conduction band. Their edges $U_{\mathrm{vb}}$ and $U_{*}$ depend on $z$ because of the negative space charge accumulated from the plasma in response to the electron and ion fluxes originating from a plasma source at $z=z_{w}$. The blue dashed curve on the left indicates the $z$-dependent edge for valence band holes as explained in more detail in the main text. The plasma side shows the electric potential energy for electrons and ions arising from the positive space charge in front of the wall. In addition the ion's ionization level $I$ at the turning point $z_{\mathrm{tp}}$ and its broadening $\Gamma$ due to the hybridization with the surface are shown. Neutralization of ions impinging on the interface leads to the injection of valence band holes in an energy interval specified by $I$ and $\Gamma$. Far away from the interface at $z=z_{b}$ the wall is a reservoir for conduction band electrons and valence band holes. In the regions around the inflection points the system is quasineutral and field-free. The bottom of the figure shows how the species' distribution functions for left and right moving particles are determined at two particular locations $z=z_{1}$ and $z=z_{2}$.

$\mathrm{SiO}_{2}$ and $\mathrm{Si}$ surfaces exposed to a two-temperature hydrogen plasma focusing on quasi-stationary density and potential profiles. A discussion of the issues which need to be resolved before the approach becomes quantitative for realistic surfaces is given in the concluding Section 4. Mathematical details interrupting the flow of the discussion are relegated to an Appendix.

\section{Kinetic theory}

\subsection{General approach}

Before we consider the simpler case of a collisionless, perfectly absorbing dielectric surface in contact with a collisionless plasma we describe in this subsection the kinetic modeling of the electronic response of a dielectric plasma interface in broader terms. A similar formulation could be worked out for a metallic plasma interface. As far as the modeling presented in this 
subsection is concerned, the main difference between metallic and dielectric plasma-facing solids is that for metals the neutralization of ions does not always lead to the injection of holes into a fully occupied valence band. Instead, if the ion's ionization energy is small enough holes are injected into the partially filled conduction band, that is, into the same band into which also electrons are injected from the plasma. In this case, only the conduction band is involved, which moreover is partially filled. The hole representation we use in the following is then no longer advantageous and the theory on the solid side of the interface has to be formulated solely in terms of distribution functions for the conduction band electrons.

We consider a planar interface at $z=0$ separating a dielectric solid residing in the $z<0$ subspace from a plasma in the $z>0$ subspace. The theoretical treatment of the interface is based on the Poisson equation and two sets of Boltzmann transport equations operating respectively in the positive and negative half-spaces and describing in total four species: electrons and ions on the plasma side and conduction band electrons and valence band holes on the wall side. We use a species index $s=e, i, *, h$ to denote electrons, ions, conduction band electrons, and valence band holes. The interface is assumed to be homogeneous in the lateral directions $x$ and $y$ so that the spatial dependence of all quantities is reduced to a dependence on $z$. For a quasi-stationary electric double layer at a homogeneous interface the distribution functions of the various species depend thus only on the spatial coordinate $z$ and the threedimensional wave vector $\vec{k}$.

Anticipating the quantum-mechanical derivation of the matching conditions for the distribution functions [33, 34 as well as a possibly iterative numerical treatment of the Boltzmann equations along the lines employed in the transport theory of semiconductor heterostructures [36, 37] we replace the set of independent variables $(z, \vec{k})$ by $(z, E, \vec{K})$ where $E$ is the total energy and $\vec{K}$ the lateral momentum. The species' distribution functions are thus written as $F_{s}(z, E, \vec{K})$.

If not stated otherwise, we give all equations in atomic units measuring energy in Rydbergs and length in Bohr radii. The zero of the energy scale is chosen to coincide with the electron affinity $\chi>0$ of the dielectric, that is, we set $E=\chi \equiv 0$.

The model on which our calculations rest is shown in Fig. 1. As it is drawn it already assumes an electric double layer with a negative and positive space charge, respectively, inside the wall and inside the plasma. The double layer is driven by a source at $z=z_{w}>0$ releasing an electron flux $j_{e}$ and an ion flux $j_{i}$. We treat only the quasi-stationary case. The fluxes are thus equal to each other and exactly balanced by loss processes inside the wall. Far away from the interface, at $z=z_{b}<0$, the wall becomes a reservoir for conduction band electrons and valence band holes.

On the wall side Fig. 1 shows a valence and a conduction band. Their edges are given by

$U_{\mathrm{vb}}(z)=-U_{c}(z)-E_{g}-\chi$,

$U_{*}(z)=-U_{c}(z)-\chi$

with $E_{g}$ the energy gap between the valence and the conduction band and $U_{c}(z)=e V_{c}(z)$ the electric potential energy given by the solution of the Poisson equation,

$\frac{d}{d z} \varepsilon(z) \frac{d}{d z} U_{c}(z)=8 \pi \rho(z)$

where

$\rho(z)=\rho_{w}(z) \theta(-z)-\rho_{p}(z) \theta(z)$

is the charge density and $\varepsilon(z)=\varepsilon \theta(-z)+\theta(z)$ is the dielectric constant, both split, with the help of the Heaviside function $\theta(z)$, into a wall and a plasma part. The connection between the solutions of the Poisson equation in the two half-spaces of the interface is given by the matching condition for the electric potential energy,

$U_{c}\left(0^{-}\right)=U_{c}\left(0^{+}\right)$and $\varepsilon \frac{d}{d z} U_{c}\left(0^{-}\right)=\frac{d}{d z} U_{c}\left(0^{+}\right)$.

The way $\rho(z)$ is defined in (4), the charge densities on the wall and the plasma side are given by

$\rho_{w}(z)=n_{*}(z)-n_{D}-n_{h}(z)+n_{A}$,

$\rho_{p}(z)=n_{i}(z)-n_{e}(z)$,

where $n_{D}$ and $n_{A}$ are, respectively, the concentration of donors and acceptors while $n_{s}(z)$ are the species's densities obtained by integrating the distribution functions over the independent variables $E$ and $\vec{K}$,

$n_{s}(z)=\int \frac{d E d^{2} K}{(2 \pi)^{3}} \frac{F_{s}(z, E, \vec{K})}{v_{s}(z, E, \vec{K})}$,

where $v_{s}(z, E, \vec{K})$ is the absolute value of the $z$-component of the species' velocity. Since the distribution functions $F_{s}$ depend via the Boltzmann equation (see below) on $U_{c}$, the Poisson equation is in general a highly nonlinear integro-differential equation.

The physical meaning of the band edges is as follows: $U_{*}(z)$ gives the lowest energy a conduction band electron can have at $z<0$. Likewise $U_{\mathrm{vb}}(z)$ gives the highest energy a valence band electron can have at location $z<0$. At the plasma-wall interface valence band electrons per se are not directly relevant. What matters are the electrons in the valence band which have been extracted from it by neutralizing an ion impinging on the interface [14. It is thus natural to describe the valence band in terms of missing electrons, that is, in terms of holes [38. Instead of using electrons 
with a negative charge and a negative effective mass the hole picture describes the valence band by quasiparticles with a positive charge and a positive effective mass. The energy a hole can have at location $z<0$ is always larger then

$U_{h}(z)=-U_{\mathrm{vb}}=U_{c}(z)+E g+\chi$,

indicated by the dashed blue line in Fig. 1. It is the edge for the motion of valence band holes.

On the plasma side the model contains ions and electrons. Their energies are given by

$U_{\mathrm{i}}(z)=U_{c}(z)$,

$U_{\mathrm{e}}(z)=-U_{c}(z)$

with $U_{c}(z)$ the solution of the Poisson equation in the positive half-space. The energies for ions and electrons at position $z>0$ are always larger than, respectively, $U_{i}(z)$ and $U_{e}(z)$. Figure 1 also shows the ionization level $I$ of the ion and its broadening $\Gamma$, both taken at the turning point $z_{\mathrm{tp}}$ of the ion trajectory. These two energies are needed in the hole injection model to be described later. The ionization energy determines at what energy the hole is injected into the valence band and the broadening gives the energy range over which injection may occur. Notice, it is not the kinetic energy of the ion's center of mass motion which matters for hole injection but the ion's internal potential energy $I$.

At the bottom of Fig. 1 we illustrate how distribution functions at particular locations, for instance, $z=z_{1}<0$ and $z=z_{2}>0$ can be determined if the distribution functions are known at the boundaries $z=z_{b}$ and $z=z_{w}$ and matching conditions are available connecting distribution functions across the interface at $z=0$. For each species $s$ we distinguish particles moving to the left from particles moving to the right. Hence, we write

$F_{s}(z, E, \vec{K})=F_{s}^{<}(z, E, \vec{K})+F_{s}^{>}(z, E, \vec{K})$

with $F_{s}^{<}$and $F_{s}^{>}$characterized, respectively, by $v_{z, s}=$ $-v_{s}<0$ and $v_{z, s}=v_{s}>0$, where $v_{s}$ is the absolute value of the $z$-component of the species' velocity. Assuming for simplicity parabolic bands inside the dielectric, the velocities can be written as

$v_{s}(z, E, \vec{K})=2\left(\frac{m_{e}}{m_{s}}\left[E-U_{s}(z)\right]-\left(\frac{m_{e}}{m_{s}} \vec{K}\right)^{2}\right)^{1 / 2}$,

where $m_{e}$ is the electron mass and $m_{s}$ is the species's (effective) mass.

Far away from the interface we assume the distribution functions to be local Maxwellians,

$\frac{F_{s}^{\mathrm{LM}}(z, E, \vec{K})}{n_{s}^{\mathrm{LM}}(z)}=\left(\frac{4 \pi m_{e}}{k_{B} T_{s} m_{s}}\right)^{3 / 2} \exp \left(-\frac{E-U_{s}(z)}{k_{B} T_{s}}\right)$

normalized to the density

$\int \frac{d E d^{2} K}{(2 \pi)^{3}} \frac{F_{s}^{\mathrm{LM}}(z, E, \vec{K})}{v_{s}(z, E, \vec{K})}=n_{s}^{\mathrm{LM}}(z)$.
The temperatures $T_{s}$ in (14) are input parameters whereas the values the densities $n_{s}^{\mathrm{LM}}(z)$ approach at the boundaries, the boundary densities at $z=z_{b}$ and $z=z_{w}$, are considered as variables. Hence, $n_{b *}=n_{*}^{\mathrm{LM}}\left(z_{b}\right), n_{b h}=n_{h}^{\mathrm{LM}}\left(z_{b}\right), n_{s e}=n_{e}^{\mathrm{LM}}\left(z_{w}\right)$, and $n_{s i}=n_{i}^{\mathrm{LM}}\left(z_{w}\right)$ have to be determined in the course of the calculation. This adjustment of the densities is required to make the plasma source consistent with the losses and the reservoir inside the wall mimicking the self-consistent response of the plasma to the wall and vice versa as it takes place in reality. On the plasma side this leads to the sheath modeling of Schwager and Birdsall [4] which has been also used in a number of particle-in-cell simulations [10, 12].

In order to determine $F_{s}^{\gtrless}$ in the way sketched at the bottom of Fig 1 we need matching conditions at $z=0$. Using the methods employed to match distribution functions across solid interfaces, originally developed by Falkovsky 34 for charge transport inside metallic surfaces, but subsequently applied also to solid heterostructures 33, 35, the distribution functions for electrons in the plasma and electrons in the conduction band of the wall are for $E>0$ at $z=0$ connected by

$$
\begin{aligned}
F_{e}^{>}(0, E, \vec{K}) & =R(E, \vec{K}) F_{e}^{<}(0, E, \vec{K}) \\
& +[1-R(E, \vec{K})] F_{*}^{>}(0, E, \vec{K}), \\
F_{*}^{<}(0, E, \vec{K}) & =R(E, \vec{K}) F_{*}^{>}(0, E, \vec{K}) \\
& +[1-R(E, \vec{K})] F_{e}^{<}(0, E, \vec{K})
\end{aligned}
$$

with

$$
R(E, \vec{K})=\left(\frac{v_{e}(0, E, \vec{K})-v_{*}(0, E, \vec{K})}{v_{e}(0, E, \vec{K})+v_{*}(0, E, \vec{K})}\right)^{2}
$$

the quantum-mechanical reflection probability for a three-dimensional potential step of height $\chi$. For energies just above the potential step $R$ is close to unity while for large $E$ it vanishes.

It should be noted that $R$ describes only the quantum-mechanical specular reflection on the potential step. Inelastic processes inside the wall which may bring the electron back to the interface, and hence also possibly back to the plasma, if it successfully passes the potential step in the reverse direction, are not included in $R$. Hence, in (16) and (17) the reflection coefficient $R$ cannot be replaced by $1-S$ with $S$ the electron sticking coefficient obtained, for instance, by the method of invariant embedding [15] because $S$ accounts for inelastic processes which in the present modeling of the interface's particle kinetics have to be incorporated into the collision integrals of the Boltzmann equations. The same holds for secondary electron emission due to impacting electrons with energies above the band gap of the wall. It also arises from inelastic processes which have to be accounted for by the collision integrals of the Boltzmann equations. Secondary electron emission due 
to Auger neutralization of ions [16] or other heavy particle collisions with the surface, on other hand, could be incorporated into the matching conditions by augmenting the right hand side of 16 by a source function $S_{e}^{>}(E, \vec{K})$ describing the probability of the surface to emit an electron with total energy $E$ and lateral momentum $\vec{K}$ because the kinetic equations we set up do not track the occupancies of the internal electronic states of the ion (or other heavy projectiles). The particular form of this function depends on the elementary surface process. It is best worked out semiempirically along the lines we use now for the resonant neutralization of ions at a surface.

The matching of the ion and hole distribution functions differs from the matching of electron distribution functions because ions cannot enter the wall and most importantly the center of mass motions of the ions and the valence band holes are not coupled via energy and momentum conservation laws. In our model ions are resonantly neutralized at the interface whereby they inject holes into the wall's valence band. Hence, the ion and hole distribution functions are for $E>E_{g}+\chi$ at $z=0$ connected by

$F_{h}^{<}(0, E, \vec{K})=F_{h}^{>}(0, E, \vec{K})+\alpha S_{h}^{<}(E, \vec{K})$,

$F_{i}^{>}(0, E, \vec{K})=(1-\alpha) F_{i}^{<}(0, E, \vec{K})$

with $S_{h}^{>}$a source function to be constructed by other means, $\alpha$ the probability for wall neutralization, which we assume here to be independent of the energy of the ion's center of mass motion. If the ions are neutralized with probability $\alpha$ less than unity some ions have the chance to come back to the plasma, having thus velocity $v_{i}$ in negative $z$-direction and contributing thereby to $F_{i}^{<}$. Only for $\alpha=1$, that is, for perfect neutralization, no ions are coming back to the plasma, as it is often assumed in the modeling of the plasma sheath.

A model for hole injection is required to complete the description of the matching condition $(19)$. The source function $S_{h}^{<}(E, \vec{K})$ is the probability to inject an hole with total energy $E$ and lateral momentum $\vec{K}$ into the valence band. It is important to realize that in 19 and $\vec{K}$ are not the total energy and lateral momentum of the impinging ion responsible for the injection. To connect the source function $S_{h}^{<}(E, \vec{K})$ with the ion distribution function at the interface, $F_{i}^{<}(0, z, E)$, we recall that the total flux of injected holes has to be identical to the total flux of impinging ions multiplied by $\alpha$. Hence, with

$j_{s}(z)=\int \frac{d E d^{2} K}{(2 \pi)^{3}}\left[F_{s}^{>}(z, E, \vec{K})-F_{s}^{<}(z, E, \vec{K})\right]$,

we obtain by using $(20)$ the condition

$$
\int \frac{d E d^{2} K}{(2 \pi)^{3}} F_{i}^{<}(0, E, \vec{K})=\int \frac{d E d^{2} K}{(2 \pi)^{3}} S_{h}^{<}(E, \vec{K}),
$$

where the integration variables on the left (right) belong to the center of mass motion of the ion (hole). To proceed one either determines $S_{h}^{<}(E, \vec{K})$ from a microscopic model for ion neutralization at a surface or one makes plausible assumptions about the overall behavior of this function. In the next section, where we discuss the simple collisionless, perfectly absorbing interface, we assume, for instance, holes to be injected with uniform probability over the relevant energy and momentum range.

The matching conditions (16), (17), (19), and 20) are essential for our approach. A comment about their impact on the distribution functions is thus in order. The distribution functions react freely to the matching conditions, that is, the values they assume at $z=0$ are determined self-consistently by the interplay of the plasma with the solid. Close to the interface the distribution functions deviate from the distribution functions far away from it in precisely such a way as it is dictated by the matching conditions. Since the matching occurs predominately in the tails of the distribution functions, that is, at high energies, where charge carriers cross the interface, collisions have enough phase space to efficiently heal the perturbation due to the interface making a merging of the solid and plasma distribution functions possible.

Let us now turn to the (quasi-stationary) Boltzmann equations to be satisfied by the distribution functions of the four species. They can be cast into the form

$$
\left[ \pm v_{s}(z, E, \vec{K}) \frac{\partial}{\partial z}+\gamma_{s}\left[F_{s^{\prime}}^{\gtrless}\right]\right] F_{s}^{\gtrless}(z, E, \vec{K})=\Phi_{s}\left[F_{s^{\prime}}^{\gtrless}\right],
$$

where $z$ is either positive or negative, depending on the species, and $\gamma_{s}\left[F_{s^{\prime}}^{\gtrless}\right]$ and $\Phi_{s}\left[F_{s^{\prime}}^{\gtrless}\right]$ are collision integrals which also depend on the species. For instance, ions may suffer charge-exchange collisions whereas electrons may be collisionless on the plasma side but subject to intra- and interband Coulomb and phonon collisions on the wall side. Similarly holes may also suffer intra- and interband Coulomb and phonon collisions. In addition, electron-hole recombination may take place inside the wall, for instance, via Auger or radiative processes. All of it has to be included in the collision integrals $\gamma_{s}$ and $\Phi_{s}$. Since in the following we numerically treat only the collisionless interface we do not give explicit formulae for $\gamma_{s}$ and $\Phi_{s}$ but they can be worked out in all cases using the techniques of semiclassical kinetic theory [39].

Once the collision integrals are specified the set of equations for the theoretical description of the electric double layer is complete. It contains the modifications of the band structure inside the dielectric wall as well as the modification of the electric potential in front of the wall due to the permanent influx of electrons and ions. The fluxes are released from a plasma source and annihilated inside the wall. How the fluxes are 
annihilated, radiatively or non-radiatively, is beyond the present model. Solving the Poisson equation(3) together with the Boltzmann equations 23) for the four species subject to the matching conditions (5), (16), (17), (19), and (20) gives the species' distribution functions and eventually the density and potential profiles of the double layer across the interface. The source and the reservoir can be made self-consistent by enforcing additional conditions to fix the boundary densities $n_{b *}, n_{b h}, n_{s e}$, and $n_{s i}$, which depend however on what kind of collisions are included. This in turn determines the way the system of Boltzmann-Poisson equations is solved numerically. In the next subsection we treat the simplest case-a collisionless interface-in full detail. It thus becomes apparent what additional conditions are needed and how the Boltzmann-Poisson equations are actually solved.

\subsection{Collisionless, perfectly absorbing surface}

So far the description of our approach was quite general. We now specialize the treatment to the simplest possible case: a collisionless plasma in contact with a perfectly absorbing collisionless surface. The reason is a practical one. In this particular case the Boltzmann equations become first order ordinary differential equations from which the two-dimensional lateral momentum $\vec{K}$ can be eliminated. Since $\vec{K}$ also drops out from the perfect absorber matching conditions, one no longer deals with distribution functions depending on four independent variables $(z, E, \vec{K})$ but only on two $(z, E)$. Without collision integrals the Boltzmann equations can be furthermore easily integrated yielding, in addition, charge densities which do not explicitly depend on $z$ but only on $U_{c}(z)$. The Poisson equation can thus be integrated once analytically and the remaining numerical task is rather modest. In Sect. 3 we will discuss how realistic the collisionless theory is.

The lateral momentum can be eliminated as follows. First, the collisionless Boltzmann equations are integrated over the lateral momentum $\vec{K}$ before switching from $(z, \vec{k})$ to $(z, E, \vec{K})$ as independent variables. They then become equations for

$F_{s}(z, k)=\int \frac{d^{2} K}{(2 \pi)^{2}} F_{s}(z, \vec{K}, k)$.

Switching then from $(z, k)$ to $(z, E)$, with $E$ now the total energy without the kinetic energy in the lateral directions, leads to collisionless Boltzmann equations of the form

$\pm v_{s}(z, E) \frac{\partial}{\partial z} F_{s}^{\gtrless}(z, E)=0$

from which the distribution functions $F_{s}^{\gtrless}(z, E)$ can be determined. Since $E$ no longer contains the kinetic energy in the lateral directions, the velocities in 25 are given by

$v_{s}(z, E)=2\left(\frac{m_{e}}{m_{s}}\left[E-U_{s}(z)\right]\right)^{1 / 2}$

with $U_{s}(z)$ still defined as before.

Next, setting $R=0$ for a perfectly absorbing surface, the matching conditions for the electron distribution functions, (16) and (17), can be transformed back to $\vec{k}$-space and integrated there over $\vec{K}$ leading to conditions for $F_{*, e}^{\gtrless}(z, k)$. Changing now the independent variables $(z, k)$ to $(z, E)$ as in the Boltzmann equations leads to conditions for $F_{*, e}^{\gtrless}(z, E)$ with $E$ again the total energy without the kinetic energy in lateral directions. Obviously, this procedure is only applicable if $R$ is assumed to be independent of $\vec{k}$. In general this is not the case. Hence, to reduce in the general matching conditions the number of independent variables requires additional assumptions about the energy and momentum dependence of $R$. Only for the perfect absorber model, where $R=0$ from the start, no further assumptions are necessary. For $\alpha=1$, as again postulated by the perfect absorber model, the matching conditions for ions can be handled similarly. Hence, the lateral momentum can be also eliminated from them.

Altogether, in terms of the functions $F_{s}^{\gtrless}(0, E)$ the matching conditions for the collisionless, perfectly absorbing interface become

$F_{e}^{>}(0, E)=F_{*}^{>}(0, E)=0$,

$F_{*}^{<}(0, E)=F_{e}^{<}(0, E)$,

$F_{h}^{<}(0, E)=F_{h}^{>}(0, E)+S_{h}^{<}(E)$,

$F_{i}^{>}(0, E)=0$.

The first equation indicates that for electrons not to come back to the plasma, as postulated by the perfect absorber model, we have to assume not only $R=0$ but in addition $F_{*}^{>}(0, E)=0$ for $E>0$. This thermalization condition is necessary because $R$ is the same in Eqs. (16) and (17). Quantum mechanical reflection by a potential step does not depend on the direction the step is crossed. Conditions (29) and (30) describe a surface perfectly annihilating ions by injecting holes into the valence band. Assuming the source function $S_{h}^{<}(E)$ to be a uniform probability for hole injection, it is given by

$S_{h}^{<}(E)=\bar{S}[\theta(E-I+\Gamma / 2)-\theta(E-I-\Gamma / 2)]$

with the normalization

$\bar{S}=\frac{1}{\Gamma} \int_{U_{w}}^{0} d E F_{i}^{<}(0, E)$

to ensure, at the interface, the equality of ion and hole flux. In the normalization condition for $S_{h}^{<}(E)$ we already anticipated for $F_{i}^{<}(0, E)$ the range of integration discussed in Fig. 2 and set $U_{w}=U_{c}\left(z_{w}\right)$. 
Equations 25 can be solved by a trajectory analysis taking matching and boundary conditions into account as illustrated in Fig. 2 . In the collisionless model the boundary conditions at $z=z_{b}$ and $z=z_{w}$ are given by the Maxwellians

$$
\frac{F_{s}^{\mathrm{LM}}(z, E)}{n_{s}^{\mathrm{LM}}(z)}=\left(\frac{4 \pi m_{e}}{k_{B} T_{s} m_{s}}\right)^{1 / 2} \exp \left(-\frac{E-U_{s}(z)}{k_{B} T_{s}}\right)
$$

as follows: At $z=z_{b}$ we enforce $F_{*, h}^{>}\left(z_{b}, E\right)=$ $F_{*, h}^{\mathrm{LM}}\left(z_{b}, E\right)$ for $E>U_{*, h}\left(z_{b}\right)$ whereas at $z=z_{w}$ we require $F_{e, i}^{<}\left(z_{w}, E\right)=F_{e, i}^{\mathrm{LM}}\left(z_{w}, E\right)$ for $E>U_{e, i}\left(z_{w}\right)$. This is illustrated in Fig. 2 by the vertical blue lines. The densities at $z=z_{b}$ and $z=z_{w}$, the boundary densities, given by $n_{b *}=n_{*}^{\mathrm{LM}}\left(z_{b}\right), n_{b h}=n_{h}^{\mathrm{LM}}\left(z_{b}\right)$, $n_{s e}=n_{e}^{\mathrm{LM}}\left(z_{w}\right)$, and $n_{s i}=n_{i}^{\mathrm{LM}}\left(z_{w}\right)$ are as pointed out in the previous section variables to be determined by the kinetic model. In addition to the boundary conditions the solution of (25) requires the matching conditions at $z=0$ symbolized in Fig. 2 by the vertical red lines. Since the trajectory analysis is standard [9] it is not explicitly given here. The principle of the calculation is described in the caption of Fig. 2 .

It turns out that the densities obtained from the solutions of (25) depend only on $U_{c}(z)$ and not on $z$ explicitly. This greatly simplifies the further processing of the densities. In particular, it allows us to obtain the first integral of the Poisson equation analytically. What remains to be done numerically is only the calculation of the second integral of the Poisson equation and the solution of four nonlinear algebraic equations. This is of course much easier than a numerical solution of a set of collisional BoltzmannPoisson equations. In our view this justifies working out the collisionless, perfectly absorbing interface. Despite its idealistic nature the numerical solution may lead to insights useful for attacking the full problem.

Let us first consider the fluxes obtained from the solutions of 25). Using the definition (21) with the obvious modifications arising from the fact that we eliminated the lateral momentum $\vec{K}$ we find

$$
\begin{aligned}
& j_{*}=j_{e}=n_{s e}\left(\frac{k_{B} T_{e}}{\pi}\right)^{1 / 2} \exp \left(-\frac{U_{w}}{k_{B} T_{e}}\right), \\
& j_{h}=j_{i}=n_{s i}\left(\frac{m_{e}}{\pi} \frac{k_{B} T_{i}}{m_{i}}\right)^{1 / 2}
\end{aligned}
$$

for the fluxes on the plasma and the wall side of the double layer. Hence, the matching conditions and the hole injection model preserve by construction the fluxes across the interface. At quasi-stationarity, the electron and ion fluxes satisfy moreover the flux balance condition,

$j_{i}=j_{e}$,

leading to a first condition involving densities at the
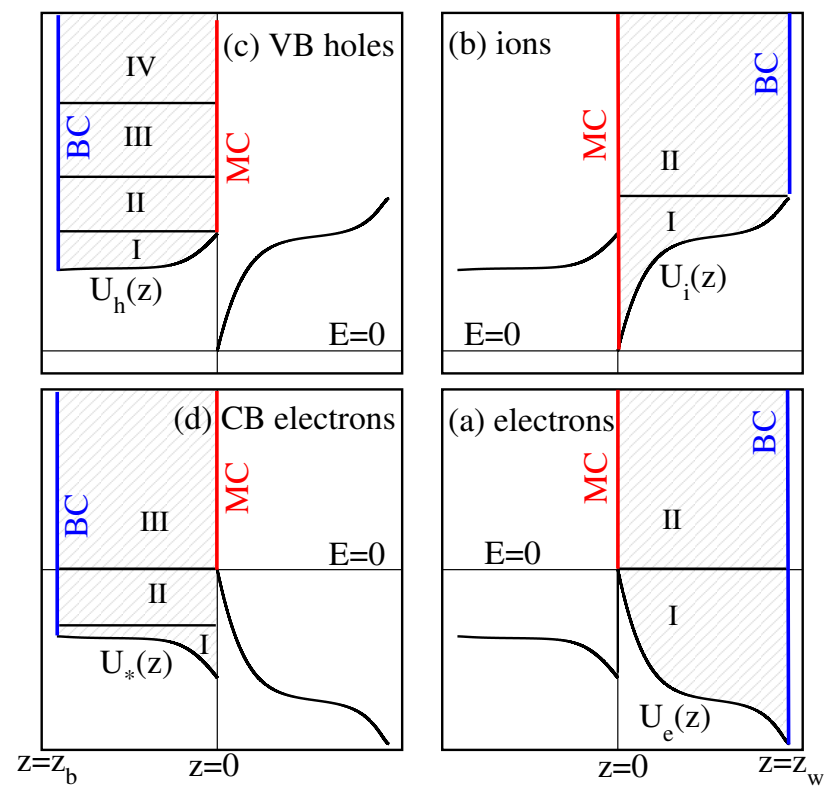

Figure 2. (Color online) The panels show the domains of quasifree motion of electrons (a), ions (b), valence band holes (c), and conduction band electrons (d) between the boundaries at $z=z_{w}$ or $z=z_{b}$, depending on the type of particle considered, and the interface at $z=0$. The boundary conditions at $z=z_{w}$ and $z=z_{b}$ and the matching conditions at $z=0$ are indicated by blue and red vertical lines. For energies where no matching conditions apply specular reflection occurs. As an example let us consider panel (a) for the electrons on the plasma side. Due to the absence of collisions the function $F_{e}^{<}(z)$ is given for $E>U_{e}$ by $F_{e}^{\mathrm{LM}}\left(z_{w}\right)$ while the function $F_{e}^{>}(z)=F_{e}^{<}(z)=F_{e}^{\mathrm{LM}}\left(z_{w}\right)$ for $E<0$ (region I) but identical to the function $F_{e}^{>}(0, E)$ given by the matching conditions at $z=0$ for $E>0$ (region II). A similar analysis applies to the other species as well. One only has to remember that regions not directly connected to the boundary conditions cannot contain any particles because of the lack of collisions. For instance, region I in panel (d) cannot be populated by conduction band electrons. Likewise region I in panel (b) is void of ions. Taking these considerations into account the solutions of 25 can be constructed.

system's boundaries

$\frac{n_{s i}}{n_{s e}}=\left(\frac{k_{B} T_{e}}{k_{B} T_{i}} \frac{m_{i}}{m_{e}}\right)^{1 / 2} \exp \left(-\frac{U_{w}}{k_{B} T_{e}}\right)$.

The densities $n_{s}(z)=n_{s}\left(U_{c}(z)\right)$ are obtained from (8) using (12), again with the obvious modifications arising from the elimination of $\vec{K}$. With the expressions for $n_{s}\left(U_{c}\right)$ given in Appendix A the source for the Poisson equation on the plasma side becomes

$\rho_{p}(z)=\rho_{p}\left(U_{c}\right)=n_{i}\left(U_{c}\right)-n_{e}\left(U_{c}\right)$

while on the wall side the source is given by

$\rho_{w}(z)=\rho_{w}\left(U_{c}\right)=\rho_{w}^{t}\left(U_{c}\right)+\rho_{w}^{j}\left(U_{c}\right)$

with

$\rho_{w}^{t}\left(U_{c}\right)=n_{*}^{t}\left(U_{c}\right)-n_{D}-n_{h}^{t}\left(U_{c}\right)+n_{A}$,

$\rho_{w}^{j}\left(U_{c}\right)=n_{*}^{j}\left(U_{c}\right)-n_{h}^{j}\left(U_{c}\right)$.

In the formulas given above, the densities $n_{*, h}^{t}\left(U_{c}\right)$ describe conduction band electrons and valence band 
holes which are thermalized/trapped within the wall while the densities $n_{*, h}^{j}\left(U_{c}\right)$ originating from 27 and (28) describe carriers coming from the continuing influx of electrons and ions to the interface after quasistationarity has been reached. This influx does not stop after the double layer is fully developed. Quasistationarity makes the electron and ion fluxes coincide, rather than vanish. In the expressions for $n_{*, h}^{j}$ we employed already (36) to replace the fluxes $j_{*}$ and $j_{h}$ by the ion density $n_{s i}$.

Since the Poisson equation's sources $\rho_{p, w}$ depend only on $U_{c}$ it can be integrated once in each half-space. Let us first consider the plasma side. Multiplying (3) by $U_{c}^{\prime}$, where the prime indicates here and in the formulae to follow a derivative with respect to $z$, and using $U_{c}\left(0^{+}\right)=0$, together with $U_{c}^{\prime}\left(z_{w}\right)=0$, which forces the double layer to be field-free at $z=z_{w}$, yields

$U_{c}^{\prime}(z)=\mathcal{F}\left(U_{c}\right)$,

where

$\mathcal{F}\left(U_{c}\right)=\left(16 \pi \int_{U_{c}}^{U_{w}} d \bar{U}_{c} \rho_{p}\left(\bar{U}_{c}\right)\right)^{1 / 2}$

with $U_{w}=U_{c}\left(z_{w}\right)$. In Appendix A the result of this integration is given. The potential profile on the plasma side can thus be obtained by integrating 42 . from $z=0$ to $z>0$. The result is

$\int_{0}^{U_{c}} \frac{d \bar{U}_{c}}{\mathcal{F}\left(\bar{U}_{c}\right)}=z$

with $0 \leq z \leq z_{w}$.

In our model we assume $\rho_{w}^{t}$ to act inside the wall as the only source for the electric potential energy $U_{c}$. It is this part of the wall's charge density which balances the positive charge density $\rho_{p}$ on the plasma side of the interface. The density $\rho_{w}^{j}$ acts in our model not as a source. Instead it will be made to vanish (physically due to electron-hole recombination inside the wall, see below). Thereby it yields an additional condition which in conjunction with the other conditions to be satisfied at the interface enables us to calculate also the continuing influx of electrons and ions. Using thus inside the wall $\rho_{w}^{t}$ as the only source for $U_{c}$ and a procedure similar to the one employed to derive 42 leads us to

$U_{c}^{\prime}(z)=\mathcal{G}^{t}\left(U_{c}\right)$,

where

$\mathcal{G}^{t}\left(U_{c}\right)=\left(\frac{16 \pi}{\varepsilon} \int_{U_{b}}^{U_{c}} d \bar{U}_{c} \rho_{w}^{t}\left(\bar{U}_{c}\right)\right)^{1 / 2}$

and $U_{b}=U_{c}\left(z_{b}\right)$. The function $\mathcal{G}^{t}\left(U_{c}\right)$ is given in Appendix A. In deriving these expressions we used $U_{c}\left(0^{-}\right)=0$ which guarantees continuity of $U_{c}$ at $z=0$, as required by the first condition in (5). In addition we forced the double layer to be field-free at $z=z_{b}$ by setting $U_{c}^{\prime}\left(z_{b}\right)=0$. The potential profile on the wall side is given by integrating (45) from $z<0$ to $z=0$ resulting in

$\int_{U_{c}}^{0} \frac{d \overline{\mathcal{G}}_{c}}{\mathcal{G}^{t}\left(\bar{U}_{c}\right)}=-z$

with $z_{b} \leq z \leq 0$.

In order to incorporate into the formalism the role we want the densities $n_{*, h}^{j}$ to play, we now take a closer look at them. As already mentioned they arise from the continuing influx of electrons and ions after the quasi-stationarity of the double layer has been reached. Hence, for a quasi-stationary double layer $n_{*, h}^{j}$ cannot act as a source for an electric field as we already anticipated in 45). It is thus reasonable to assume that they recombine nearby the interface, perhaps in a spatial zone stretching from $z=0$ to $z=z_{1}<0$, where $z_{1}$ is a distance from the interface not yet specified. Below we will assume $z_{1}$ to coincide with the inflection point of $U_{c}(z)$ inside the wall which we need to match the double layer to a quasi-neutral, field-free region, as it happens in reality. On the plasma side an inflection point has to be implemented for the same reason. There it is required to match the sheath to a quasi-neutral, field-free plasma [4.

In view of what we just said, we hence postulate the recombination condition,

$\int_{z_{1}}^{0} d z \rho_{w}^{j}(z)=\int_{U_{1}}^{0} d U_{c} \frac{\rho_{w}^{j}\left(U_{c}\right)}{\mathcal{G}^{t}\left(U_{c}\right)}=0$

with $U_{1}=U_{c}\left(z_{1}\right)$ the electric potential energy at the inflection point $z=z_{1}$. In the second equality we used (45) to replace the $z$-integration by an integration over $U_{c}$. The last condition we finally have to enforce is the jump condition for the derivative of the electric potential energy at $z=0$ as stated in (5). Using (42) and (45) it becomes

$\varepsilon \mathcal{G}^{t}(0)=\mathcal{F}(0)$

with $\mathcal{F}\left(U_{c}\right)$ and $\mathcal{G}^{t}\left(U_{c}\right)$ defined in 43 and 46 .

Now, we have all the ingredients together to formulate a self-consistent model for the electric double layer at a collisionless, perfectly absorbing plasma-wall interface.

Towards that end let us first discuss the necessity of implementing inflection points into the potential profile $U_{c}(z)$. It can be most clearly seen by setting hypothetically $n_{s i}=n_{s e}$ and $n_{b *}=n_{b h}$, that is, by making the source and the reservoir charge-neutral. Such a choice would however not lead to $\rho_{p}\left(z_{w}\right)=0$ and $\rho_{w}\left(z_{b}\right)=0$ as one would perhaps naively expect. Hence, by initially assuming at $z=z_{b}$ and $z=z_{w}$ distribution functions $F_{s}^{\mathrm{LM}}$ confronts us at the end with charge non-neutral boundaries if at the same time we force the net charge of the boundary densities to be zero. Hence, $F_{s}^{\mathrm{LM}}$ cannot describe the quasineutral regions into which the double layer should be embedded. 
The reason is of course that some particles are, depending on their energy and/or type, either absorbed or emitted by the interface. Hence, they are lost from or gained by a half-space of the double layer preventing thereby $F_{s}^{\gtrless}$ to re-establish $F_{s}^{\mathrm{LM}}$ at the boundaries. In reality the distribution functions react self-consistently to the presence of the interface making thereby the double layer also charge-neutral far away from the interface. By postulating the form of the distribution functions at $z=z_{b}$ and $z=z_{w}$ we destroyed this mechanism. Alternatively we could have enforced charge-neutrality at the boundaries. But then we could not have known the distribution functions making the solution of 25) much more complicated.

To mimic the self-consistent reaction of the distribution functions far away from the interface we follow Schwager and Birdsall [4] and consider the boundary densities, $n_{b *}, n_{b h}, n_{s e}$, and $n_{s i}$, appearing in $F_{s}^{\mathrm{LM}}$ at $z=z_{b}$ and $z=z_{w}$, respectively, as variables to be determined from the calculation. This requires to incorporate two inflection points into the potential profile, one at $z=z_{1}<0$ inside the wall and one at $z=z_{p}>0$ inside the plasma. The conditions for the inflection points are charge-neutrality

$\rho_{w}^{t}\left(U_{1}\right)=0$

$\rho_{p}\left(U_{p}\right)=0$

and the vanishing of the electric field

$\mathcal{G}^{t}\left(U_{1}\right)=0$,

$\mathcal{F}\left(U_{p}\right)=0$

with $U_{1}=U_{c}\left(z_{1}\right)$ and $U_{p}=U_{c}\left(z_{p}\right)$. Notice, on the wall side only $\mathcal{G}^{t}$ appears.

The charge-neutrality conditions (50) and 51 have to be specified further. On the plasma side it translates simply into

$n_{e}\left(U_{p}\right)=n_{i}\left(U_{p}\right)$.

On the wall side, however, charge-neutrality is more involved since in addition to (50) we also have to satisfy

$n_{*}^{t}\left(U_{1}\right) n_{h}^{t}\left(U_{1}\right)=n_{\mathrm{i}}^{2}$

with

$n_{\mathrm{i}}=\frac{1}{4}\left(\frac{k_{B} T_{*}}{\pi}\right)^{3 / 2}\left(\frac{m_{*} m_{h}}{m_{e}^{2}}\right)^{3 / 4} \exp \left(-\frac{E_{g}}{2 k_{B} T_{*}}\right)$

the intrinsic charge density 26 of the wall at temperature $T_{*}=T_{h}$. Using the formulae for the densities given in Appendix A and solving Eqs. (50) and (55) simultaneously, assuming either $n_{A}=n_{D}=0$ (intrinsic wall), $n_{A}=0$ (n-doped wall), or $n_{D}=0$ (pdoped wall) we obtain two conditions for the boundary densities $n_{b *}$ and $n_{b h}$ :

$$
\frac{n_{b *}}{n_{\text {ref }}}=N_{*}^{2} \frac{\exp \left(\frac{U_{b}-U_{1}}{k_{B} T_{*}}\right)}{\Phi\left(\sqrt{\frac{U_{1}+\chi}{k_{B} T_{*}}}\right)-\Phi\left(\sqrt{\frac{U_{1}-U_{b}}{k_{B} T_{*}}}\right)}
$$

and

$\frac{n_{b h}}{n_{\text {ref }}}=N_{h}^{2} \exp \left(\frac{U_{1}-U_{b}}{k_{B} T_{h}}\right)$,

where $\Phi(\sqrt{y})$ is the error function, see Appendix A For an intrinsic wall $n_{\text {ref }}=n_{\text {int }}$ and $N_{*}=N_{h}=1$, for a p-type wall $n_{\text {ref }}=n_{p}=\left[n_{A}+\sqrt{n_{A}^{2}+4 n_{i}^{2}}\right] / 2$ leading to $N_{*}=2 x /\left(1+\sqrt{1+4 x^{2}}\right)$ and $N_{h}=1$ with $x=n_{\text {int }} / n_{A}$, while for a n-type wall $n_{\text {ref }}=$ $n_{n}=\left[n_{D}+\sqrt{n_{D}^{2}+4 n_{i}^{2}}\right] / 2$ yielding $N_{*}=1$ and $N_{h}=2 x /\left(1+\sqrt{1+4 x^{2}}\right)$ with $x=n_{\text {int }} / n_{D}$. From the jump condition (49) we finally obtain an equation relating $n_{s i} / n_{\text {ref }}$ to the density ratios $n_{b *} / n_{\text {ref }}$ and $n_{b h} / n_{\text {ref }}$ making the approach self-consistent.

The description of our approach is now complete. The modeling we propose for a quasi-stationary electric double layer at a collisionless, perfectly absorbing plasma-wall interface contains eight parameters: Four energies $U_{b}, U_{1}, U_{p}$, and $U_{w}$ and four densities $n_{b *}$, $n_{b h}, n_{s e}$, and $n_{s i}$. Eight equations are available to determine them: The three conditions (37), (57), and (58) for the boundary densities, the quasi-neutrality condition on the plasma side (54), the two conditions forcing the double layer to be field-free around $z=z_{1}$ and $z=z_{p},(52),(53)$, the recombination condition (48) and the jump condition (49) guaranteeing at the end that the double layer is globally charge neutral between its physically relevant boundaries $z_{1}$ and $z_{p}$. It should be noticed that the wall provides an absolute scale via the reference density $n_{\text {ref }}$ and the band structure parameters $E_{g}$ and $\chi$, as does the ionization energy $I$ and its broadening $\Gamma$. The approach produces thus absolute numbers.

\section{Results}

In this section we use parameters applicable to $\mathrm{Si}$ and $\mathrm{SiO}_{2}$ surfaces in contact with a two-temperature hydrogen plasma to obtain numerical results for the electric double layer forming at a collisionless, perfectly absorbing plasma-wall interface. Before discussing the results we give some details about the numerical treatment of the equations derived in the previous section.

For the numerics we normalized energies on both sides of the interface to the thermal energy $k_{B} T_{e}$ of the electrons emitted from the plasma source. Lengths, in contrast, are normalized, depending on which side of the interface is considered, to the electron Debye length of the wall,

$\lambda_{D}^{w}=\sqrt{\frac{\varepsilon k_{B} T_{*}}{8 \pi n_{\mathrm{ref}}}}$,

or the electron Debye length of the plasma

$\lambda_{D}^{p}=\sqrt{\frac{k_{B} T_{e}}{8 \pi n_{s e}}}$. 


\begin{tabular}{|l||llllll|}
\hline Wall & $\frac{m_{e}}{m_{h}}$ & $\begin{array}{l}k_{B} T_{*} \\
(\mathrm{eV})\end{array}$ & $\begin{array}{l}k_{B} T_{h} \\
(\mathrm{eV})\end{array}$ & $\begin{array}{l}\chi \\
(\mathrm{eV})\end{array}$ & $\begin{array}{l}E_{g} \\
(\mathrm{eV})\end{array}$ & $\varepsilon$ \\
\hline $\mathrm{Si}$ & 1.0 & 0.2 & 0.2 & 4 & 1.0 & 12 \\
$\mathrm{SiO}_{2}$ & 1.0 & 0.2 & 0.2 & 1.0 & 9.0 & 4 \\
\hline \multirow{3}{*}{ Plasma } & & & & & & \\
& $\frac{m_{e}}{m_{i}}$ & $k_{B} T_{e}$ & $k_{B} T_{i}$ & $I$ & $\Gamma$ & \\
$\left(10^{-4}\right)$ & $(\mathrm{eV})$ & $(\mathrm{eV})$ & $(\mathrm{eV})$ & $(\mathrm{eV})$ & \\
\hline $\mathrm{H}^{+}-\mathrm{e}$ & 5.4 & 2.0 & 0.2 & 13.6 & 2.0 & \\
\hline
\end{tabular}

Table 1. Material parameters for the wall and the plasma used in the numerical calculations. In order not to overload the model we neglect the image shift of the ions' ionization level. It would depend on yet another parameter, the position of the image plane. The mass of the conduction band electrons $m_{*}$ is not included in the table because it is varied between physically reasonable bounds. It should be also kept in mind that for an actual surface the parameters may deviate from the given values due to materials science aspects not addressed in this work.

After rewriting the equations in normalized form we replace the boundary density ratios $n_{s i} / n_{s e}, n_{b *} / n_{\text {ref }}$, and $n_{b h} / n_{\text {ref }}$ by (37), (57), and (58). We then obtain four equations for the four (normalized) potential energy drops $y_{1}=U_{1} / k_{B} T_{e}, y_{b}=U_{b} / k_{B} T_{e}, y_{p}=$ $U_{p} / k_{B} T_{e}$, and $y_{w}=U_{w} / k_{B} T_{e}$. They factorize into two sets of two equations each, one for $\left(y_{p}, y_{w}\right)$ and one for $\left(y_{b}, y_{1}\right)$.

More specifically, the equations for $\left(y_{p}, y_{w}\right)$ arise from the quasi-neutrality condition (54) and the fieldfree condition (53). After replacing the boundary ratio $n_{s i} / n_{s e}$ by (37) they become nonlinear equations for $\left(y_{p}, y_{w}\right)$ alone and can be casted into the form

$f_{\mathrm{I}}\left(y_{p}, y_{w}\right)=0$

$f_{\mathrm{II}}\left(y_{p}, y_{w}\right)=0$.

Except for the difference arising from the different choice of the zero of the energy axis these two equations are identical to the ones given by Schwager and Birdsall [4. Replacing the boundary density ratios $n_{b *} / n_{\text {ref }}$ and $n_{b h} / n_{\text {ref }}$ by (57) and (58) in the field-free condition (52) and the recombination condition (48) leads to two nonlinear equations for $\left(y_{1}, y_{b}\right)$. They can be casted in the same form,

$f_{\mathrm{III}}\left(y_{1}, y_{b}\right)=0$

$f_{\mathrm{IV}}\left(y_{1}, y_{b}\right)=0$.

The nonlinear equations (61)- 64 contain no additional physical information. They are therefore not explicitly given.

We solve 61) -64 graphically as explained in Fig. 3 below. The solutions $\left(y_{p}, y_{w}\right)$ on the plasma side and $\left(y_{1}, y_{b}\right)$ on the wall side are linked to each other by the jump condition for the electric field 449 which in normalized form becomes a condition containing all four boundary density ratios. Since the boundary ratios $n_{b *} / n_{\text {ref }}, n_{b h} / n_{\text {ref }}$, and $n_{s i} / n_{s e}$ are known from the potential drops the fourth ratio $n_{s i} / n_{\text {ref }}$ can be
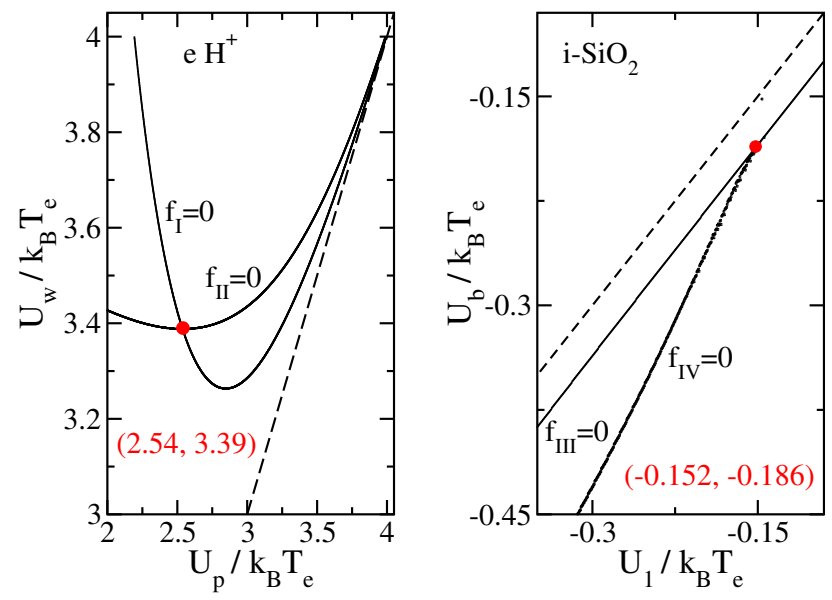

Figure 3. (Color online) The left panel shows the solutions of $f_{\mathrm{I}}\left(y_{p}, y_{w}\right)=0$ and $f_{\mathrm{II}}\left(y_{p}, y_{w}\right)=0$. Where the two curves cross, indicated by the red bullet, gives the normalized potential drops $\left(y_{p}, y_{w}\right)=(2.54,3.39)$. With $k_{B} T_{e}=2 \mathrm{eV}$ follows $U_{p}=5.08 \mathrm{eV}$ and $U_{w}=6.78 \mathrm{eV}$ with $U_{p}$ the potential of the collector sheath. In the right panel the graphical solution of $f_{\mathrm{III}}\left(y_{1}, y_{b}\right)=0$ and $f_{\mathrm{IV}}\left(y_{1}, y_{b}\right)=0$ is shown leading to the normalized potential drops $\left(y_{1}, y_{p}\right)=(-0.152,-0.186)$, again marked by the red bullet. Hence, $U_{1}=-0.304 \mathrm{eV}$ and $U_{b}=-0.372 \mathrm{eV}$. The potential drop $U_{1}$ is the band bending inside the wall. The effective mass of the conduction band electrons is $m_{*}=m_{e} / 1.3$. Plasma parameters and the remaining wall parameters are given in Table 1

determined from (49) making thereby the wall side of the double layer consistent with the plasma side. At this point the recombination condition (48) turned out to be essential. Without it the collisionless theory had not enough equations to determine all the unknown parameters.

For the numerical calculations we take the parameters given in Table 1. A few comments about this choice are in order. The thermal energy of the electrons released from the plasma source is $k_{B} T_{e}=$ $2 \mathrm{eV}$. For the ions we take $k_{B} T_{i}=0.2 \mathrm{eV}$. The ion temperature is thus rather high but decreasing it further produced numerical instabilities already on the plasma side. Since it is reasonable to assume the ions to set the lowest thermal energy for the charged species, we also set $k_{B} T_{*}=k_{B} T_{h}=0.2 \mathrm{eV}$. For the small band gap material $\mathrm{Si}$ the intrinsic charge density (56) is then rather high, leading to unrealistically high densities in the double layer. But making the reservoir colder than the coldest species of the sources seems to be physically not plausible and might have made the numerical solution of $f_{\mathrm{III}}=f_{\mathrm{IV}}=0$ even more subtle than it turned out to be already. The masses we take are the ones for a hydrogen plasma and a $\mathrm{Si}$ or a $\mathrm{SiO}_{2}$ surface. In the energy range where we need the (effective) masses of the wall's charge carriers they are a bit uncertain [40, 41, 42. We used therefore the mass of the conduction band electrons as a parameter to be varied within physically reasonable bounds. 


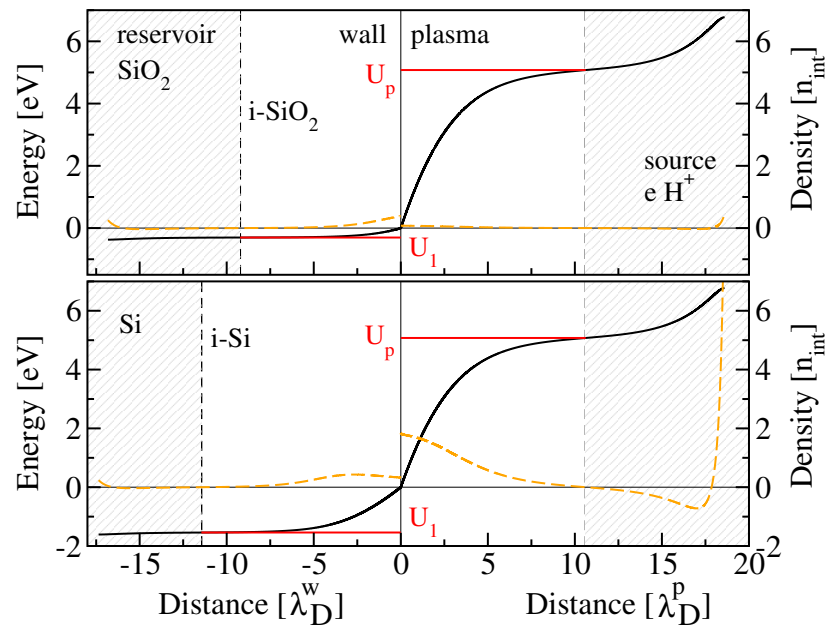

Figure 4. (Color online) Potential profile for $\mathrm{i}_{-} \mathrm{SiO}_{2}$ (upper panel) and i-Si (lower panel) in contact with a hydrogen plasma with the properties specified in Table 1 The black curves are the potential profiles in $\mathrm{eV}$ (left ordinate) and the orange curves give the density profiles $\rho_{p}(z)$ and $\rho_{w}^{t}(z)$ as defined in 38 and (40) in units of $n_{\text {int }}$ (right ordinate). The grey regions indicate respectively the reservoir and the source which have been set up to provide the correct physical boundaries for the double layer. Relevant for the double layer is thus only the region between the vertical dashed lines giving the position of the inflection points $z_{1}$ and $z_{p}$ in units of the electron Debye screening lengths $\lambda_{D}^{w}$ and $\lambda_{D}^{p}$, respectively. Hence, the sheath potential is given by $U_{p}$ and the band bending by $U_{1}$. The mass of the conduction band electrons is $m_{*}=m_{e} / 1.3$ for $\mathrm{SiO}_{2}$ and $m_{*}=m_{e} / 2$ for $\mathrm{Si}$. The remaining surface parameters can be found in Table 1 together with the plasma parameters.

As mentioned, to determine the simultaneous roots of $f_{\mathrm{I}}\left(y_{p}, y_{w}\right)=0$ and $f_{\mathrm{II}}\left(y_{p}, y_{w}\right)=0$ on the one hand and $f_{\mathrm{III}}\left(y_{1}, y_{b}\right)=0$ and $f_{\mathrm{IV}}\left(y_{1}, y_{b}\right)=0$ on the other we used a graphical approach. First, we determined from $f_{\mathrm{I}}\left(y_{p}, y_{w}\right)=0$ and $f_{\mathrm{II}}\left(y_{p}, y_{w}\right)=$ 0 two separate relations for $y_{w}\left(y_{p}\right)$. Plotting them and looking for points where they cross gives the simultaneous root $\left(y_{p}, y_{w}\right)$ as shown in the left panel of Fig. 3. A similar procedure for $f_{\mathrm{III}}\left(y_{1}, y_{b}\right)=0$ and $f_{\mathrm{IV}}\left(y_{1}, y_{b}\right)=0$, depicted in the right panel, gives the root $\left(y_{1}, y_{b}\right)$. On the plasma side the equations turned out to be more handy than on the wall side as can be seen by the noisiness of the roots of $f_{\mathrm{IV}}$. Nevertheless in all the cases we discuss in this work the simultaneous solutions of the nonlinear equations (63) and (64) are stable and reproducible. After the normalized energies $y_{b}, y_{1}, y_{p}$, and $y_{w}$ are known, the density ratios $n_{b *} / n_{\text {ref }}, n_{b h} / n_{\text {ref }}, n_{s i} / n_{s e}$ and $n_{s i} / n_{\text {ref }}$ can be determined. All the parameters we introduced in the modeling of the double layer are then fixed and the potential profile $U_{c}(z)$ can be calculated by integrating (44) and (47).

Using $m_{*}=m_{e} / 1.3$ and $m_{*}=m_{e} / 2$, respectively, for the masses of the conduction band electrons in i$\mathrm{SiO}_{2}$ and i-Si, which we consider most reasonable for
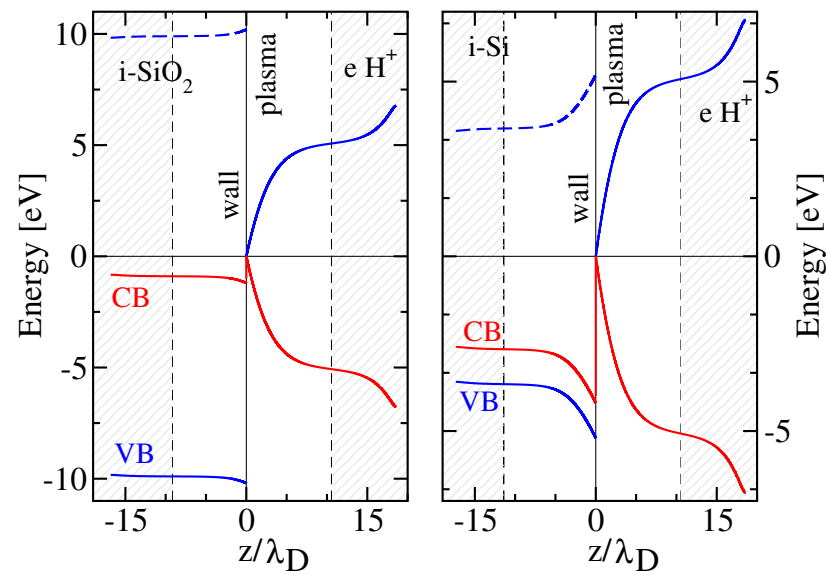

Figure 5. (Color online) Band edges for $\mathrm{i}-\mathrm{SiO}_{2}$ (left panel) and i-Si (right panel) in contact with a hydrogen plasma with the parameters given in Table 1 Inside the wall the solid red (blue) curves are the edges for the conduction (valence) band and in front of the wall the red (blue) curves give the potential energy for electrons (ions). The dashed blue lines are the edges for the valence band holes. Lengths are given in units of the electron Debye screening lengths $\lambda_{D}^{w}$ on the wall side and $\lambda_{D}^{p}$ on the plasma side. For $\mathrm{SiO}_{2}$ the effective mass for the conduction band electrons is $m_{*}=m_{e} / 1.3$ while for $\mathrm{Si}$ it is $m_{*}=m_{e} / 2$. The other parameters can be found in Table 1

the elevated temperatures [40, 42, we plot in Fig. 4 the potential profiles (black curves) together with the profiles for the charge density (orange curves). The density profiles show that the source as well as the reservoir are not charge-neutral as expected. Chargeneutrality is satisfied by construction only around the inflection points $z=z_{1}$ and $z=z_{p}$. It is interesting to note that for i-Si the density of the space charge increases not monotonously towards the wall. We attribute this to the unrealistically high intrinsic charge concentration $n_{\text {int }}$ arising from the high temperature. The band gap in $\mathrm{Si}$ is only around $1 \mathrm{eV}$ and thus only five times larger than the thermal energy of the charge carriers. The potential drops on the wall and the plasma side relevant for the double layer are $U_{1}$ and $U_{p}$. The former is the plasma-induced band bending whereas the latter is the wall-induced sheath potential. The potential drops from $z=z_{w}$ to $z=z_{p}$ and from $z=z_{1}$ to $z=z_{b}$ on the other hand are required to establish the Maxwellians at the boundaries. The flatness around the inflection points depends on the accuracy with which the roots $\left(y_{b}, y_{1}\right)$ and $\left(y_{p}, y_{w}\right)$ have been determined. Increasing the accuracy makes the plateaus wider but the differences $y_{1}-y_{b}$ and $y_{w}-y_{p}$ remain the same.

The potential profile $U_{c}(z)$ determines via (1) and (2) the edges of the bands inside the wall. For i$\mathrm{SiO}_{2}$ and $\mathrm{i}-\mathrm{Si}$ this is shown in Fig. 5 together with the potential energies in front of the wall. The mass of the conduction band electrons is again $m_{*}=m_{e} / 1.3$ 
for $\mathrm{SiO}_{2}$ and $m_{*}=m_{e} / 2$ for $\mathrm{Si}$. From the discussion of Fig. 4 it is clear that in the grey regions the boundary conditions are established. Hence, only the profiles between the grey regions apply directly to the double layer. The figure gives an idea how strong a hydrogen plasma with the parameters specified in Table 11 modifies the band structure of $\mathrm{i}-\mathrm{SiO}_{2}$ and i-Si. For n- and p-type $\mathrm{SiO}_{2}$ and $\mathrm{Si}$ surfaces the band structure would look qualitatively similar. The absolute numbers, however, would be different because the reference densities $n_{\text {ref }}$ are different leading for instance to different electron Debye screening lengths. Instead of plotting the band structures for different dopings we summarize representative results for doped surfaces in Table A1. The surfaces are always in contact with the hydrogen plasma specified in Table1.

Besides the band bending $U_{1}$ and the sheath potential $U_{p}$ Table A1 contains also data for the net electron and ion flux $j=j_{e}=j_{i}$ towards the interface and the charge density $N_{\mathrm{EDL}}$ in one leg of the double layer,

$N_{\mathrm{EDL}}=\int_{z_{1}}^{0} d z \rho_{w}^{t}(z)=\int_{0}^{z_{p}} d z \rho_{p}(z)$.

Since between $z=z_{1}$ and $z=z_{p}$ the double layer is charge neutral, the charge density confined inside the wall between $z=z_{1}$ and $z=0$ is by construction equal to the charge density between $z=0$ and $z=$ $z_{p}$ on the plasma side. Numerically the integrated charge densities coincide better than one percent. The intrinsic charge density $n_{\text {int }}$ depends according to (56) on the mass of the conduction band electrons. Varying $m_{*}$ changes thus even for fixed $x=\infty$ (intrinsic), $x=n_{\text {int }} / n_{A}$ (p-type) and $x=n_{\text {int }} / n_{D}$ (n-type) the reference density $n_{\text {ref }}=n_{\text {int }}$ (intrinsic), $n_{\text {ref }}=n_{p}$ (ptype), and $n_{\text {ref }}=n_{n}$ (n-type) and thus the boundary condition to be met by the double layer on the wall side. As a result, the properties of the double layer depend on $m_{*}$ as can be seen in Table A1.

For Si surfaces the fluxes $j$ and the charge densities $N_{\text {EDL }}$ trapped in one leg of the double layer are rather high. The reason is the high ion temperature required to stabilize the numerical calculations. Since the ion temperature sets also the scale for the temperature of the charge carriers inside the wall, the intrinsic density $n_{\text {int }}$, and with it the reference densities $n_{\text {ref }}$, are very high for small band gap materials such as $\mathrm{Si}$. The unrealistically high densities of the reservoir lead also to unrealistically high densities in the plasma source. As a result, the electron Debye screening lengths $\lambda_{D}^{w, p}$ are extremely short making the space charges on both sides of the interface very narrow, at most $100 \AA$ wide. For any realistic gas pressure the double layer would be thus collisionless on the plasma side. It would be even almost collisionless on the solid side, because the inelastic scattering length $l_{\text {inel }} \approx 100 \AA$ [25]. For
$\mathrm{Si}: \mathrm{H}^{+}$-e the premises of our numerical calculations are thus satisfied but only for a situation which in practice cannot be realized.

The data for $\mathrm{SiO}_{2}: \mathrm{H}^{+}-\mathrm{e}$ are more realistic because the band gap of $\mathrm{SiO}_{2}$ is significantly larger than the thermal energy of the reservoir leading to reasonable reference densities. The fluxes $j$ and the charge densities $N_{\text {EDL }}$ are then also more realistic. Due to the lower densities the electron Debye screening lengths are much larger leading to wider space charge layers. On the plasma side, the space charge can still be considered collisionless because, based on $l_{\mathrm{cx}}=$ $1 / n_{g} \sigma_{\mathrm{cx}}=k_{B} T_{g} / \sigma_{\mathrm{cx}} p$ with $\sigma_{\mathrm{cx}} \approx 10^{-15} \mathrm{~cm}^{2}$ [2] the cross section for charge-exchange scattering and $k_{B} T_{g}=0.03 \mathrm{eV}$ the gas temperature, the chargeexchange scattering length $l_{\mathrm{cx}}$ will be much larger than $10^{-3} \mathrm{~cm}$ for any reasonable gas pressure $p$. On the solid side, however, collisions cannot be ignored anymore because the space charge is now much wider than the inelastic scattering length $l_{\text {inel }} \approx 100 \AA$ [25]. It should be however recalled that the recombination condition (48) anticipates interband collisions. There is thus some hope that the data presented for $\mathrm{SiO}_{2}$ give the correct order of magnitude. But only an investigation which includes intra- and interband collisions inside the wall, and thus requires on the solid side the numerical solution of the collisional Boltzmann equations (to be considered beyond the scope of the present work), can tell whether this is indeed the case.

The band bending we find for $\mathrm{i}_{-} \mathrm{SiO}_{2}$ in contact with a hydrogen plasma with $k_{B} T_{e}=2 \mathrm{eV}, k_{B} T_{i}=$ $0.2 \mathrm{eV}$, and an ion density $n_{s i} \approx 1.3 \cdot 10^{11} \mathrm{~cm}^{-3}$ (which follows from the numerical values for $n_{s i} / n_{\text {ref }}$ and $n_{\text {ref }}$ given in Table A1) is about $0.3 \mathrm{eV}$. In our previous work 32 we obtained for a helium plasma with $k_{B} T_{e}=2 \mathrm{eV}, k_{B} T_{i}=0$, and a plasma density $n_{0}=10^{7} \mathrm{~cm}^{-3}$ a band bending of about $0.1 \mathrm{eV}$. In view of the much denser plasma considered in the present work the larger band bending we find now is to be expected. Thus, the numbers we obtain are consistent with our previous work. In contrast to it, however, the new approach presented in this paper, is more general because it does not dependent on a thermodynamical principle. Working with Boltzmann equations and matching conditions for the distribution functions gives us more flexibility in treating ions, electrons, valence band holes, and conduction band electrons. In addition, it is conceivable by using time-dependent Boltzmann equations to study within this framework also the temporal build-up of the double layer. Time-dependent Boltzmann equations would be also required for tracking the time-periodic modifications of the band structure induced by radio-frequency (micro)discharges. The approach we presented in this paper could be also applied to this problem. 


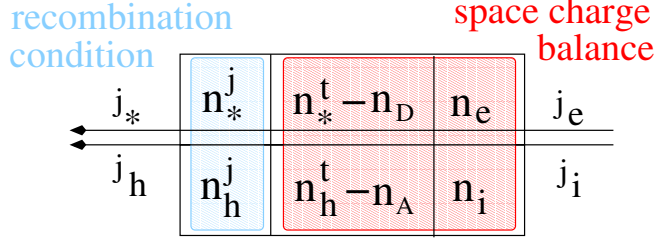

Figure 6. (Color online) Quintessence of our kinetic approach for modeling a quasi-stationary electric double layer at a plasmafacing solid. The net positive space charge in the plasma sheath $\rho_{p}(z)=n_{i}(z)-n_{e}(z)$ is balanced by the thermalized/trapped net negative space charge inside the wall $\rho_{w}^{t}(z)=n_{*}^{t}(z)-n_{D}-$ $n_{h}^{t}(z)+n_{A}$. The non-thermalized charges $\rho_{w}^{j}(z)=n_{*}^{j}(z)-n_{h}^{j}(z)$ inside the wall, arising from the continuing influx of electrons and ions recombine and limit thereby in conjunction with the matching and boundary conditions the fluxes $j_{e}$ and $j_{i}$ released from the plasma source. By extending the modeling into the wall and distinguishing in it thermalized/trapped from nonthermalized charge carriers the equality of electron and ion fluxes can be reconciled with a vanishing ion density at the interface because not the ions matter directly but the holes in the valence band.

\section{Conclusions}

We presented a semiclassical kinetic theory for the quasi-stationary electric double layer at a planar dielectric surface in contact with a two-temperature plasma. It is based on the Poisson equation for the electric potential energy and two sets of Boltzmann equations, operating in disjunct half-spaces, for the species involved in the electronic response of the plasma and the solid: electrons and ions on the plasma side and conduction band electrons and valence band holes on the wall side. Crucial for the kinetic theory are the matching conditions for the distribution functions across the interface. For electrons they are identical to the ones employed in solid heterostructures whereas for the ion and hole distribution functions they represent a hole injection model. As boundary conditions far away from the interface we use Maxwellian distribution functions. The species' boundary densities as well as the potential drops in the double layer are determined in the course of the calculation which at the end yields self-consistent potential and density profiles across the interface.

The physical picture implemented in our approach is that of a quasi-stationary electric double layer sandwiched between quasi-neutral, field-free regions. On the plasma side this region mimics the bulk plasma whereas on the wall side it mimics either an intrinsic or an extrinsic dielectric wall. To enforce these physical boundary conditions a reservoir for conduction band electrons and valence band holes has to be attached to the wall side while on the plasma side an electron and ion source is required. The reservoir and the source per se have no direct physical meaning. They are only technical devices to implement the physical boundary conditions.

Figure 6 summarizes the essence of our approach. The net electron and ion fluxes originating from the plasma source lead to a double layer consisting of electrons and ions on the plasma side and thermalized as well as non-thermalized conduction band electrons and valence band holes on the wall side. The net positive space charge of the double layer on the plasma side is balanced inside the wall only by the thermalized net negative space charge. Recombination of the wall's non-thermalized charge densities (which in the collisionless theory is taken into account by an ad-hoc recombination condition) limits in conjunction with the matching and boundary conditions the net electron and ion influx after the double layer is established. Inside the wall the ion flux becomes a flux of valence band holes, whereas the flux of electrons becomes a flux of conduction band electrons. At quasi-stationarity these two fluxes have to give rise to a permanent flux of photons and/or phonons depending on whether the recombination occurs radiatively or non-radiatively. This is however not yet included in the modeling.

Applying the kinetic approach to a collisionless, perfectly absorbing plasma-wall interface with material parameters representing $\mathrm{Si}$ and $\mathrm{SiO}_{2}$ surfaces in contact with a hydrogen plasma demonstrated its feasibility. Since the wall sets an absolute scale for energy and density we could determine absolute numbers for the potential drops, the charge densities on either side of the double layer, and the particle fluxes maintaining the double layer. In view of the high ion temperature necessary to stabilize the numerics on the plasma side, which in turn implied a high temperature for the charge carriers inside the wall, the numerical data for the small band gap material Si turned out to be unrealistic. For the large band gap material $\mathrm{SiO}_{2}$, however, we obtained rather reasonable results showing that the kinetic approach presented in this work has the potential to describe the electric response of a real surface to a real plasma and vice versa if it is applied to a more realistic model of the plasma-wall interface. Not only collisions and quantum-mechanical reflection from the interface have then to be included but also a realistic description of the wall's band structure.

Obviously for a realistic interface model the kinetic equations become rather complex, even in the quasi-stationary regime. Iterative solution strategies have then to go hand-in-hand with Monte-Carlo techniques to tackle the problem. It may be also required to reduce the complexity by a systematic multiple-time scale analysis, anticipating the different time scales on which intra- and interband scattering processes occur, which we expect to separate the loss processes due the recombination of conduction band electrons with valence band holes from the 
thermalizing collisions. The ad-hoc recombination condition we employed in the modeling of the collisionless, perfectly absorbing interface may then turn out to be a secular condition in a multiple timescale analysis. We leave this conjecture for future investigations.

No matter how powerful the techniques for attacking the kinetic equations, the quality of the results depends on the quality of the electronic band structure. Even in the absence of the uncertainties arising from plasma-induced imperfections and chemical contaminations, getting all the required band structure information is a challenge. Since electrons and holes are injected into states far away from the band edges, knowing the band structure in the vicinity of the band gap is not sufficient. The dispersion of the conduction (valence) band has to be known all the way up (down) to the electron affinity (ionization level of the ions in the plasma). To restrict the considerations to two bands with one energy valley may in fact be unjustified as well as the effective mass approximation. The kinetic modeling of the electronic response of a realistic plasma-wall interface has thus to go hand in hand with advanced surface diagnostics and ab-initio band structure calculations.

\section{Acknowledgments}

Support from the Deutsche Forschungsgemeinschaft through Project B10 of the Transregional Collaborative Research Center SFB/TRR 24 is greatly acknowledged.

\section{Appendix A. Mathematical technicalities}

In this appendix we give for the collisionless, perfectly absorbing plasma-wall interface the formulae for the charge densities $n_{s}\left(U_{c}\right)$ with $s=e, i, *, h$ and the integrated charge densities $\mathcal{F}_{e, i}\left(U_{c}\right)$ and $\mathcal{G}_{*, h}^{t}\left(U_{c}\right)$ from which

$$
\begin{aligned}
& \mathcal{F}\left(U_{c}\right)=\sqrt{\left[\mathcal{F}_{i}\left(U_{c}\right)\right]^{2}-\left[\mathcal{F}_{e}\left(U_{c}\right)\right]^{2}}, \\
& \mathcal{G}^{t}\left(U_{c}\right)=\sqrt{\left[\mathcal{G}_{*}^{t}\left(U_{c}\right)\right]^{2}-\left[\mathcal{G}_{h}^{t}\left(U_{c}\right)\right]^{2}}
\end{aligned}
$$

follow. These functions can be obtained, as described in Sect. 2, from the solutions of the collisionless Boltzmann equations 25) constructed from the trajectory analysis explained in Fig. 2 .

On the plasma side the charge densities are

$$
\begin{aligned}
& n_{i}\left(U_{c}\right)=\frac{n_{s i}}{2} \exp \left(\frac{U_{w}-U_{c}}{k_{B} T_{i}}\right) \Phi_{c}\left(\sqrt{\frac{U_{w}-U_{c}}{k_{B} T_{i}}}\right), \\
& n_{e}\left(U_{c}\right)=\frac{n_{s e}}{2} \exp \left(\frac{U_{c}-U_{w}}{k_{B} T_{e}}\right)\left[1+\Phi\left(\sqrt{\frac{U_{c}}{k_{B} T_{e}}}\right)\right]
\end{aligned}
$$

On the wall side we distinguish thermalized from non-thermalized charge carriers. The density of conduction band electrons is therefore given by

$n_{*}\left(U_{c}\right)=n_{*}^{t}\left(U_{c}\right)+n_{*}^{j}\left(U_{c}\right)$

with

$$
\begin{aligned}
n_{*}^{t}\left(U_{c}\right) & =n_{b *} \exp \left(\frac{U_{c}-U_{b}}{k_{B} T_{*}}\right)\left[\Phi\left(\sqrt{\frac{U_{c}+\chi}{k_{B} T_{*}}}\right)\right. \\
& \left.-\Phi\left(\sqrt{\frac{U_{c}-U_{b}}{k_{B} T_{*}}}\right)\right]
\end{aligned}
$$

the density of the thermalized conduction band electrons and

$$
\begin{aligned}
n_{*}^{j}\left(U_{c}\right) & =n_{s i}\left(\frac{m_{*}}{4 m_{i}} \frac{k_{B} T_{i}}{k_{B} T_{e}}\right)^{1 / 2} \exp \left(\frac{\chi+U_{c}}{k_{B} T_{e}}\right) \\
& \times \Phi_{c}\left(\sqrt{\frac{\chi+U_{c}}{k_{B} T_{e}}}\right)
\end{aligned}
$$

the density of the non-thermalized conduction band electrons.

Similarly, the density of valence band holes is written as

$n_{h}\left(U_{c}\right)=n_{h}^{t}\left(U_{c}\right)+n_{h}^{j}\left(U_{c}\right)$

with

$n_{h}^{t}\left(U_{c}\right)=n_{b h} \exp \left(\frac{U_{b}-U_{c}}{k_{B} T_{h}}\right)$

the thermalized density. The non-thermalized density reads

$n_{h}^{j}\left(U_{c}\right)=n_{s i}\left(\frac{m_{h}}{m_{i}} \frac{k_{B} T_{i}}{\pi}\right)^{1 / 2} \frac{\Psi\left(U_{c}\right)}{2 \Gamma}$

with

$\Psi\left(U_{c}\right)=2\left(\sqrt{A-U_{c}}-\sqrt{B-U_{c}}\right)$

and $A=I+(\Gamma / 2)-E_{g}-\chi$ and $B=I-(\Gamma / 2)-E_{g}-\chi$. The particular form of $\Psi\left(U_{c}\right)$ is the result of the uniform injection probability employed in the hole injection model.

The integrated densities, the Sagdeev-type potentials [28, 29, 30] in our double layer theory, are

$$
\begin{aligned}
{\left[\mathcal{F}_{i}\left(U_{c}\right)\right]^{2} } & =8 \pi n_{s i} k_{B} T_{i}\left[\exp \left(\frac{U_{w}-U_{c}}{k_{B} T_{i}}\right)-1\right. \\
& \left.-H\left(\frac{U_{w}-U_{c}}{k_{B} T_{i}}, 0\right)\right]
\end{aligned}
$$

for the ions,

$$
\begin{aligned}
{\left[\mathcal{F}_{e}\left(U_{c}\right)\right]^{2} } & =8 \pi n_{s e} k_{B} T_{e}\left[1-\exp \left(\frac{U_{c}-U_{w}}{k_{B} T_{e}}\right)\right. \\
& \left.+\exp \left(-\frac{U_{w}}{k_{B} T_{e}}\right) H\left(\frac{U_{w}}{k_{B} T_{e}}, \frac{U_{c}}{k_{B} T_{e}}\right)\right]
\end{aligned}
$$


for the electrons,

$$
\begin{aligned}
{\left[\mathcal{G}_{*}^{t}\left(U_{c}\right)\right]^{2} } & =\frac{16 \pi}{\varepsilon} n_{* b} k_{B} T_{*}\left[\exp \left(-\frac{U_{b}+\chi}{k_{B} T_{*}}\right)\right. \\
& \left.\times H\left(\frac{U_{c}+\chi}{k_{B} T_{*}}, \frac{U_{b}+\chi}{k_{B} T_{*}}\right)-H\left(\frac{U_{c}-U_{b}}{k_{B} T_{*}}, 0\right)\right] \\
& -\frac{16 \pi}{\varepsilon} n_{D}\left(U_{c}-U_{b}\right)
\end{aligned}
$$

for thermalized conduction band electrons and

$$
\begin{aligned}
{\left[\mathcal{G}_{h}^{t}\left(U_{c}\right)\right]^{2} } & =\frac{16 \pi}{\varepsilon} n_{h b} k_{B} T_{h}\left[1-\exp \left(\frac{U_{b}-U_{c}}{k_{B} T_{h}}\right)\right] \\
& -\frac{16 \pi}{\varepsilon} n_{A}\left(U_{c}-U_{b}\right)
\end{aligned}
$$

for thermalized valence band holes.

The function

$$
\begin{aligned}
H(a, b)=\int_{a}^{b} d y e^{y} \Phi(\sqrt{y}) & =e^{b} \Phi(\sqrt{b})-e^{a} \Phi(\sqrt{a}) \\
& -\sqrt{\frac{4 b}{\pi}}+\sqrt{\frac{4 a}{\pi}}
\end{aligned}
$$

is an auxiliary function and

$\Phi(\sqrt{y})=\frac{1}{\sqrt{\pi}} \int_{0}^{y} d x \frac{e^{-x}}{\sqrt{x}}$

is the error function. As usual the function $\Phi_{c}(\sqrt{y})$ appearing in A.3 is the complementary error function.

\section{References}

[1] Franklin R N 1976 Plasma phenomena in gas discharges (Oxford: Clarendon Press)

[2] Lieberman M A and Lichtenberg A J 2005 Principles of plasma discharges and materials processing (New York: Wiley-Interscience)

[3] Langmuir I and Mott-Smith H 1924 Gen. Electr. Rev. 27 449

[4] Schwager L A and Birdsall C K 1990 Phys. Fluids B 21057

[5] Riemann K U 1991 J. Phys. D: Appl. Phys. 24493

[6] Franklin R N 2003 J. Phys. D: Appl. Phys. 36 R309

[7] Brinkmann R P 2009 J. Phys. D: Appl. Phys. 42194009

[8] Robertson S 2013 Plasma Phys. Control. Fusion 55093001

[9] $\mathrm{Hu} \mathrm{P} \mathrm{N}$ and Ziering S 1966 Phys. Fluids 92168

[10] Taccogna F, Longo S and Capitelli M 2004 Phys. Plasmas 111220

[11] Sydorenko D, Kaganovich I, Raitses Y and Smolyakov A 2009 Phys. Rev. Lett. 103145004

[12] Gyergyek T and Kovacic J 2012 Phys. Plasmas 19013506

[13] Campanell M D 2015 Physics of Plasmas 22040702

[14] Marbach J, Bronold F X and Fehske H 2012 Eur. Phys. J. D 66106

[15] Bronold F X and Fehske H 2015 Phys. Rev. Lett. 115 225001

[16] Phelps A V and Petrović Z L 1999 Plasma Sources Sci. Technol. 8 R21

[17] Demidov V I, Adams S F, Kaganovich I D, Koepke M E and Kurlyandskaya I P 2015 Phys. Plasma 22104501

[18] Schoenbach K H and Becker K 2016 Eur. Phys. J. D 7029

[19] Kushner M J 2004 J. Appl. Phys. 95846

[20] Tchertchian P A, Wagner C J, Houlahan T J, Li B, Sievers D J and Eden J G 2011 Contrib. Plasma Phys. 51889

[21] Ostrom N P and Eden J G 2005 Appl. Phys. Lett. 87141101
[22] Dussart R, Overzet L J, Lefaucheux P, Dufour T, Kulsreshath M, Mandra M A, Tillocher T, Aubry O, Dozias S, Ranson P, Lee J B and Goeckner M 2010 Eur. Phys. J. D 60601

[23] Kulsreshath M K, Schwaederle L, Overzet L J, Lefaucheux $\mathrm{P}$, Ladroue J, Tillocher $\mathrm{T}$, Aubry $\mathrm{O}$, Woytasik $\mathrm{M}$, Schelcher G and Dussart R 2012 J. Phys. D: Appl. Phys. 45285202

[24] Eden J G, Park S J, Cho J H, Kim M H, Houlahan T J, Li B, Kim E S, Kim T L, Lee S K, Kim K S, Yoon J K, Sung S H, Sun P, Herring C M and Wagner C J 2013 IEEE Trans. Plasma Sci. 41661

[25] H Lüth 2015 Solid Surfaces, Interfaces and Thin Films (Berlin: Springer)

[26] Li S S 2006 Semiconductor Physical Electronics (New York: Springer)

[27] Andrews J G and Allen J E 1971 Proc. Roy. Soc. Lond. A 320459

[28] Schamel H and Bujarbarua S 1983 Phys. Fluids 26190

[29] Raadu M A 1989 Phys. Reports 17825

[30] Charles C 2007 Plasma Sources Sci. Technol. 16 R1

[31] Tkharev E E and Danilyuk A L 1985 Vacuum 35183

[32] Heinisch R L, Bronold F X and Fehske H 2012 Phys. Rev. $B \mathbf{8 5} 075323$

[33] Dugaev V K, Litvinov V I and Petrov P P 1995 Phys. Rev. $B \mathbf{5 2} 5306$

[34] Falkovsky L A 1983 Advances in Physics 32753

[35] Schroeder D 1992 J. Appl. Phys. 72964

[36] Baranger H U and Wilkins J W 1987 Phys. Rev. B 361487

[37] Denis A R S and Pulfrey D L 1998 J. Appl. Phys. 844959

[38] Hess K 1988 Advanced theory of semiconductor devices (Englewood Cliffs: Prentice Hall)

[39] Smith H and Jensen H 1989 Transport phenomena (Oxford: Clarendon Press)

[40] van Driel H M 1984 Appl. Phys. Lett. 44617

[41] Evtukh A A 2001 Ukr. J. Phys. 461087

[42] Riffe D M 2002 J. Opt. Soc. Am. B 191092 


\begin{tabular}{|c|c|c|c|c|c|c|c|c|c|c|c|c|}
\hline Wall & $\mathrm{x}$ & $\frac{m_{e}}{m_{*}}$ & $\begin{array}{l}n_{\mathrm{ref}} \\
\left(10^{18}\right. \\
\left.\mathrm{cm}^{-3}\right)\end{array}$ & $\begin{array}{l}\lambda_{D}^{w} \\
\left(10^{-8}\right. \\
\mathrm{cm})\end{array}$ & $\begin{array}{l}\lambda_{D}^{p} \\
\left(10^{-8}\right. \\
\mathrm{cm})\end{array}$ & $\frac{n_{b *}}{n_{\text {ref }}}$ & $\frac{n_{b h}}{n_{\mathrm{ref}}}$ & $\frac{n_{s i}}{n_{\text {ref }}}$ & $\begin{array}{l}U_{1} \\
(\mathrm{eV})\end{array}$ & $\begin{array}{l}U_{b} \\
(\mathrm{eV})\end{array}$ & $\begin{array}{l}N_{\mathrm{EDL}} \\
\left(10^{12}\right. \\
\left.\mathrm{cm}^{-2}\right)\end{array}$ & $\begin{array}{l}j \\
\left(10^{24}\right. \\
\left.\mathrm{s}^{-1} \mathrm{~cm}^{-2}\right)\end{array}$ \\
\hline \multirow[t]{2}{*}{ p-Si } & 0.6 & 2.0 & 56.28 & 15.35 & 3.36 & 0.26 & 1.03 & 80.11 & -1.46 & -1.47 & 28.21 & 129.14 \\
\hline & & 1.5 & 70.02 & 13.78 & 6.5 & 0.26 & 1.03 & 17.23 & -0.45 & -0.46 & 14.5 & 33.34 \\
\hline \multirow[t]{2}{*}{ i-Si } & $\infty$ & 2.0 & 26.32 & 22.43 & 6.69 & 1.75 & 1.42 & 43 & -1.59 & -1.66 & 14.28 & 31.7 \\
\hline & & 1.5 & 32.73 & 20.14 & 11.61 & 1.75 & 1.42 & 11.52 & -0.5 & -0.57 & 8.32 & 10.43 \\
\hline \multirow[t]{3}{*}{ n-Si } & 1.7 & 2.0 & 35.09 & 19.40 & 18.18 & 2.12 & 1.16 & 4.36 & -1.76 & -1.91 & 5.0 & 4.23 \\
\hline & & 1.5 & 43.73 & 17.42 & 17.58 & 2.13 & 1.17 & 3.76 & -0.52 & -0.67 & 5.61 & 4.54 \\
\hline & & & $\begin{array}{l}\left(10^{10}\right. \\
\left.\mathrm{cm}^{-3}\right)\end{array}$ & $\begin{array}{l}\left(10^{-3}\right. \\
\mathrm{cm})\end{array}$ & $\begin{array}{l}\left(10^{-3}\right. \\
\mathrm{cm})\end{array}$ & & & & $(\mathrm{eV})$ & $(\mathrm{eV})$ & $\begin{array}{l}\left(10^{7}\right. \\
\left.\mathrm{cm}^{-2}\right)\end{array}$ & $\begin{array}{l}\left(10^{15}\right. \\
\left.\mathrm{s}^{-1} \mathrm{~cm}^{-2}\right)\end{array}$ \\
\hline $\mathrm{p}-\mathrm{SiO}_{2}$ & 0.6 & 1.3 & 12.15 & 1.91 & 4.15 & 0.51 & 1.08 & 2.44 & -0.29 & -0.30 & 22.96 & 8.22 \\
\hline $\mathrm{i}-\mathrm{SiO}_{2}$ & $\infty$ & & 7.49 & 2.43 & 6.21 & 1.76 & 1.42 & 1.76 & -0.30 & -0.37 & 15.07 & 3.66 \\
\hline $\mathrm{n}-\mathrm{SiO}_{2}$ & 1.7 & & 9.99 & 2.1 & 8.7 & 2.16 & 1.18 & 0.67 & -0.32 & -0.47 & 11.21 & 1.85 \\
\hline
\end{tabular}

Table A1. Numerical data for intrinsic and extrinsic $\mathrm{SiO}_{2}$ and Si surfaces exposed to the hydrogen plasma specified in Table 1 